\title{
LIGHT-ELEMENT ABUNDANCE VARIATIONS AT LOW METALLICITY: THE GLOBULAR CLUSTER NGC 5466*
}

\author{
Matthew Shetrone ${ }^{1}$, Sarah L. Martell ${ }^{2}$, Rachel Wilkerson ${ }^{3,8}$, Joshua Adams $^{4}$, Michael H. Siegel ${ }^{5}$, \\ Graeme H. SMith ${ }^{6}$, AND Howard E. BOND ${ }^{7}$ \\ ${ }^{1}$ McDonald Observatory, University of Texas at Austin, HC75 Box 1337-MCD, Fort Davis, TX 79734, USA; shetrone@ astro.as.utexas.edu \\ ${ }^{2}$ Astronomisches Rechen-Institut, Zentrum für Astronomie der Universität Heidelberg, Mönchhofstr. 12-14, D-69120 Heidelberg, Germany; \\ martell@ari.uni-heidelberg.de \\ ${ }^{3}$ Center for Astrophysics, Space Physics and Engineering Research One Baylor Place 97310, Baylor University Waco, TX 76798-7310, USA; rachelw52@gmail.com \\ ${ }^{4}$ Astronomy Department, University of Texas at Austin, Austin, TX 78712, USA; jjadams@ astro.as.utexas.edu \\ ${ }^{5}$ Department of Astronomy and Astrophysics, Pennsylvania State University, 525 Davey Laboratory, State College, PA 16801, USA; siegel@astro.psu.edu \\ ${ }^{6}$ University of California Observatories/Lick Observatory, Department of Astronomy \& Astrophysics, UC Santa Cruz, 1156 High St., Santa Cruz, CA 95064, USA; \\ graeme@ucolick.org \\ ${ }^{7}$ Space Telescope Science Institute, 3700 San Martin Dr., Baltimore, MD 21218, USA; bond@ @stsci.edu \\ Received 2010 June 18; accepted 2010 August 14; published 2010 September 10
}

\begin{abstract}
We present low-resolution $(R \simeq 850)$ spectra for 67 asymptotic giant branch (AGB), horizontal branch, and red giant branch (RGB) stars in the low-metallicity globular cluster NGC 5466, taken with the VIRUS-P integral-field spectrograph at the $2.7 \mathrm{~m}$ Harlan J. Smith telescope at McDonald Observatory. Sixty-six stars are confirmed, and one rejected, as cluster members based on radial velocity, which we measure to an accuracy of $16 \mathrm{~km} \mathrm{~s}^{-1}$ via template-matching techniques. $\mathrm{CN}$ and $\mathrm{CH}$ band strengths have been measured for 29 RGB and AGB stars in NGC 5466, and the band-strength indices measured from VIRUS-P data show close agreement with those measured from Keck/LRIS spectra previously taken for five of our target stars. We also determine carbon abundances from comparisons with synthetic spectra. The RGB stars in our data set cover a range in absolute $V$ magnitude from +2 to -3 , which permits us to study the rate of carbon depletion on the giant branch as well as the point of its onset. The data show a clear decline in carbon abundance with rising luminosity above the luminosity function "bump" on the giant branch, and also a subdued range in $\mathrm{CN}$ band strength, suggesting ongoing internal mixing in individual stars but minor or no primordial star-to-star variation in light-element abundances.
\end{abstract}

Key words: globular clusters: general - globular clusters: individual (NGC 5466) - stars: abundances

\section{INTRODUCTION}

In all Galactic globular clusters with moderate metallicity $([\mathrm{Fe} / \mathrm{H}] \simeq-1.5)$, there are star-to-star variations in the strength of the $3883 \AA \mathrm{CN}$ absorption band at any given evolutionary phase. These $\mathrm{CN}$ band strengths anticorrelate with $\mathrm{CH}$ band strengths, so that stars with weak $\mathrm{CH}$ bands (and therefore relatively low carbon abundances) have strong $\mathrm{CN}$ bands (and therefore relatively high nitrogen abundances). Band-strength variations were first observed in spectra of red giants in globular clusters (e.g., Norris \& Freeman 1979; Suntzeff 1981), but are also found in subgiants and on the main sequence (e.g., Briley et al. 2004), indicating that they are a result of primordial enrichment rather than an evolutionary effect.

Variations in the broad $\mathrm{CN}$ and $\mathrm{CH}$ features are just the most readily observed part of a larger light-element abundance pattern, a division of globular cluster stars into one group with typical Population II abundances and another that is relatively enhanced in nitrogen, sodium, and aluminum, and depleted in carbon, oxygen, and magnesium. These abundance divisions are usually studied in the anticorrelated pairs of carbon and nitrogen or oxygen and sodium (see, e.g., Kraft 1994; Gratton et al. 2004; Carretta et al. 2009). The magnesium-aluminum anticorrelation is more murky: although some researchers find

\footnotetext{
* Based in part on data obtained at the W. M. Keck Observatory, which is operated as a scientific partnership among the California Institute of

Technology, the University of California, and the National Aeronautics and Space Administration. The Observatory was made possible by the generous financial support of the W. M. Keck Foundation.

8 REU student at McDonald Observatory.
}

$\mathrm{Mg}-\mathrm{Al}$ anticorrelations in individual globular clusters (e.g., Shetrone 1996; Gratton et al. 2001), the abundance distributions are not as clearly bimodal as in the case of nitrogen, and the origin of $\mathrm{Mg}$-poor, $\mathrm{Al}$-rich stars is more difficult to fit into the general framework of enrichment by moderate-mass AGB stars (e.g., D'Ercole et al. 2008).

This paper presents a study of the behavior of $\mathrm{CN}$ and $\mathrm{CH}$ band strengths and $[\mathrm{C} / \mathrm{Fe}]$ abundances in red giant stars in the low-metallicity globular cluster NGC 5466, which has $[\mathrm{Fe} / \mathrm{H}] \simeq 2.2$ (Harris 1996, 2003 revision). Studies based on $\mathrm{CN}$ and $\mathrm{CH}$ band strength are more difficult in low-metallicity clusters, where the $\mathrm{CN}$ variation visible in the spectra can be quite small despite variations in $[\mathrm{N} / \mathrm{Fe}]$ abundance as large as in higher-metallicity globular clusters (e.g., M53, Martell et al. 2008a; M55, Briley et al. 1993). The globular clusters with metallicities similar to NGC 5466 in which light-element abundance inhomogeneities have been most extensively studied are M92 and M15. The red giants in both objects exhibit a progressive decline in $[\mathrm{C} / \mathrm{Fe}]$ with advancing evolution above the magnitude level of the horizontal branch (HB; Carbon et al. 1982; Trefzger et al. 1983; Langer et al. 1986; Bellman et al. 2001). Giants in both clusters typically exhibit weak $\lambda 3883$ CN bands (Carbon et al. 1982; Trefzger et al. 1983), and do not exhibit bimodal $\mathrm{CN}$ distributions like those in more metal-rich clusters, although a handful of stars with enhanced CN have been discovered in M15 (Langer et al. 1992; Lee 2000). Despite their lack of $\mathrm{CN}$-strong giants, both $\mathrm{M} 92$ and $\mathrm{M} 15$ exhibit anticorrelated $\mathrm{O}-\mathrm{N}, \mathrm{O}-\mathrm{Na}$ or $\mathrm{O}-\mathrm{Al}$ variations, or correlated $\mathrm{N}-\mathrm{Na}$, of the type that are commonplace in globular clusters (Norris \& Pilachowski 1985; Sneden et al. 1991, 1997; 
Shetrone 1996). In addition, Cohen et al. (2005) discovered that star-to-star differences in $[\mathrm{C} / \mathrm{Fe}]$ and $[\mathrm{N} / \mathrm{Fe}]$ abundances exist among stars near the base of the red giant branch (RGB) in $\mathrm{M} 15$, and the $\mathrm{C}$ and $\mathrm{N}$ abundances tend to be anticorrelated. These efforts have shown that for very metal-poor stars the use of $\mathrm{CN}$ to determine which stars carry the anticorrelated light-element abundance pattern can be problematic, and that anticorrelated variations in light-element abundances do exist in low-metallicity globular clusters despite their lack of obvious $\mathrm{CN}$ band-strength variation.

If NGC 5466 does contain the same primordial variations in light-element abundances as are found in higher-metallicity globular clusters, the resulting range in $\mathrm{CN}$ and $\mathrm{CH}$ band strength will be muted by the low overall metallicity of the cluster, perhaps to a degree where it is difficult to distinguish a bimodal but closely spaced $\mathrm{CN}$ band-strength distribution from a broad but unimodal distribution. If NGC 5466 does not contain primordial abundance variations, that would imply that primordial enrichment is not a universal process in low-metallicity globular clusters the way it is in higher-metallicity clusters. A metallicity limit on primordial light-element enrichment offers some insight into the primordial enrichment process, and into the larger and still-developing picture of chemical complexity within individual globular clusters (e.g., Piotto 2008).

\section{OBSERVATIONS AND REDUCTION}

We determined photometry for members of NGC 5466 based on CCD frames obtained by H.E.B. with the Kitt Peak National Observatory $0.9 \mathrm{~m}$ telescope on 1997 May 9. The T2KA chip at the Ritchey-Chretien focus provides a $23^{\prime} \times 23^{\prime}$ field, which encloses most of the cluster, which has a tidal radius of $34^{\prime}$, according to Harris (1996). We used $u B V I$ filters, as defined by Bond (2005), with exposure times of $600,45,45$, and $60 \mathrm{~s}$, respectively, under photometric conditions. We calibrated the photometry to the network of $u B V I$ standard stars established by Siegel \& Bond (2005). Data were reduced using the IRAF CCDPROC pipeline and photometry was measured with DAOPHOT/ ALLSTAR (Stetson 1987, 1994). DAOGROW (Stetson 1990) was used to perform curve-of-growth fitting for aperture correction on both program and standard stars. The raw photometry was calibrated using the iterative matrix inversion technique described in Siegel et al. (2002) to translate the photometry to the standard system of Landolt (1992) and Siegel \& Bond (2005). Table 1 lists identification numbers, positions, and photometry for the stars observed with VIRUS-P. The star identification numbers and coordinates are from an unpublished photometric catalog developed by M.H.S. from the analysis described above. The typical errors on the $B, V$, and $I$ photometry are $0.030,0.022$, and $0.032 \mathrm{mag}$, respectively.

On 2009 March 3-5 and July 28-29, we observed four fields in NGC 5466 for a total of $22 \mathrm{hr}$ with the Visible Integral-field Replicable Unit Spectrograph prototype (VIRUS-P, Hill et al. 2008) on the $2.7 \mathrm{~m}$ Harlan J. Smith Telescope at McDonald Observatory. VIRUS-P is a spectrograph with an integral-field unit (IFU) of 246 fibers in a hexagonal, one-third fill factor, close pack pattern. The fibers have a projected diameter of 4". 1 and have centers astrometrically calibrated to $0{ }^{\prime} .7$ relative to a fixed, offset guiding camera via open cluster observations. The field of view for the IFU is 2.89 square arcmin and with a set of three 7". 14 dithers the filling factor is $100 \%$. The VP1 grating was used, giving an instrumental FWHM between 5 and $6 \AA$ over the wavelength range of $3500-5800 \AA$. Arc lamp calibration produced a wavelength solution with an rms accuracy of $0.05 \AA$. Our procedure during observing was to dither the telescope and take three $1200 \mathrm{~s}$ exposures per field to fill in the spaces between the IFU fibers. Three of the fields had four sets of dithers, but the July observing run was less successful due to weather and only a single dither set was taken for the final field.

A custom software pipeline developed for the instrument by J. Adams (2010, in preparation) was used in all reductions. The notable features are that no interpolation was performed on the data to avoid correlated noise and that background subtraction was done between differently sampled fibers by fitting B-spline models (Dierckx 1993) in a running boxcar of 31 chip-adjacent fibers in a manner similar to optimal long-slit sky subtraction methods (Kelson 2003). The NGC 5466 pointing was sparse enough to allow self-sky-subtraction. The spectra were flux calibrated by observing a single flux standard on each night using the same dithering pattern used for the science targets. The flux solution was then applied to every fiber in every exposure for that night. Based on many tens of flux standards taken during other VIRUS-P projects the absolute flux calibration precision is $8.5 \%$ and wavelength independent for full dither sets. The data reduction code does not correct for differential atmospheric refraction (DAR) because there is very little impact from DAR with these large fibers when they are summed over full dither sets. Fiber spectra were then combined using the IRAF task scombine to form a single spectrum for each star. For some stars, this was done by combining a single fiber from all visits (usually four) to a specific dither position; for other stars, those falling near the edge of a fiber, it may have required combining different fibers on different dithers (perhaps 8 or 12 spectra).

Figure 1 is a color-magnitude diagram for NGC 5466, with different symbols denoting various parts of our data set: stars not observed in this particular VIRUS-P pointing are small points, and the spectroscopic sample (unblended asymptotic giant branch (AGB) and RGB stars with high enough signal to noise for index measurements) are shown as filled squares. Our standard for a spectroscopically unblended star is that there are no other stars within 5 arcsec down to 3 mag below the sample star's B magnitude. Open squares represent stars with sufficient signal-to-noise ratio $(\mathrm{S} / \mathrm{N})$ for spectroscopic analysis but possible spectroscopic blends, and open triangles represent $\mathrm{HB}$ stars and $\mathrm{RGB}$ stars with low $\mathrm{S} / \mathrm{N}$. One stronglined radial velocity non-member (star 1980) is shown as an inverted triangle. The two filled squares outlined with large circles are stars 1398 and 2483, and are likely to be AGB stars based on their positions to the blue of the giant branch. The filled square outlined with a large star is star 1839, which has strong $\mathrm{CN}$ and $\mathrm{CH}$ features, and is likely a $\mathrm{CH}$ star. Although 1839 lies on the giant branch in the $(V, V-I)$ color-magnitude diagram, the strong absorption in the UV, due to extra $\mathrm{CN}$ opacity and the Bond-Neff effect (Bond \& Neff 1969), makes its $(B-V)$ color distinctly red relative to the giant branch. The distance modulus and reddening for NGC 5466 are $(m-M)_{V}=16.15$ and $E(B-$ $V)=0.023$ (Dotter et al. 2010).

Four typical spectra are shown in Figure 2 to provide a sense for the quality and characteristics of the data. The top three spectra are a series in luminosity spanning the range of our data set, with the faintest at the top. The lowest spectrum is of star 1839 and shows the unusually strong $\mathrm{CN}$ and $\mathrm{CH}$ bands.

\subsection{The LRIS Comparison Sample}

Spectra were acquired for six RGB stars in NGC 5466 by GHS in 2003, using the LRIS double spectrograph (Oke et al. 1995) 
Table 1

Velocity Sample

\begin{tabular}{|c|c|c|c|c|c|c|c|}
\hline ID & 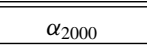 & $\delta_{2000}$ & 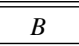 & 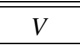 & 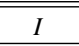 & RV $\left(\mathrm{km} \mathrm{s}^{-1}\right)$ & $\overline{\overline{\sigma_{\mathrm{RV}}\left(\mathrm{km} \mathrm{s}^{-1}\right)}}$ \\
\hline \multicolumn{8}{|c|}{ NGC 5466 members } \\
\hline 3209 & $14: 05: 23.45$ & $28: 30: 01.8$ & 16.84 & 16.51 & 16.03 & 86.5 & 2.6 \\
\hline 1409 & $14: 05: 24.49$ & $28: 32: 44.7$ & 16.47 & 16.17 & 15.74 & 87.9 & 6.1 \\
\hline 2176 & $14: 05: 25.76$ & $28: 31: 44.6$ & 17.01 & 16.60 & 16.12 & 107.9 & 11.3 \\
\hline 2481 & $14: 05: 26.39$ & $28: 31: 20.3$ & 15.52 & 14.55 & 13.47 & 112.7 & 8.2 \\
\hline 1765 & $14: 05: 27.62$ & $28: 32: 17.7$ & 16.75 & 16.61 & 16.52 & 100.1 & 2.7 \\
\hline 2245 & $14: 05: 28.05$ & $28: 31: 39.7$ & 16.78 & 16.61 & 16.39 & 105.6 & 9.5 \\
\hline 1578 & $14: 05: 29.62$ & $28: 32: 33.0$ & 16.75 & 16.68 & 16.56 & 103.9 & 4.6 \\
\hline 1899 & $14: 05: 30.42$ & $28: 32: 08.5$ & 16.78 & 16.39 & 15.87 & 81.8 & 13.7 \\
\hline 2281 & $14: 05: 31.92$ & $28: 31: 37.8$ & 17.16 & 16.40 & 15.54 & 103.7 & 3.7 \\
\hline 1524 & $14: 05: 34.48$ & $28: 32: 39.1$ & 17.16 & 16.74 & 16.20 & 93.4 & 4.0 \\
\hline 1235 & $14: 05: 36.93$ & $28: 33: 05.7$ & 16.92 & 16.11 & 15.20 & 110.8 & 9.4 \\
\hline 2753 & $14: 05: 19.35$ & $28: 30: 54.9$ & 17.85 & 17.13 & 16.33 & 151.8 & 7.6 \\
\hline 2840 & $14: 05: 24.90$ & $28: 30: 48.0$ & 18.01 & 17.31 & 16.47 & 97.1 & 10.6 \\
\hline 2113 & $14: 05: 26.18$ & $28: 31: 49.8$ & 16.96 & 16.44 & 15.73 & 130.2 & 11.5 \\
\hline 1483 & $14: 05: 30.45$ & $28: 32: 40.8$ & 16.90 & 16.49 & 15.90 & 145.3 & 6.2 \\
\hline 3305 & $14: 05: 20.71$ & $28: 29: 41.8$ & 15.67 & 14.67 & 13.58 & 128.7 & 3.5 \\
\hline 3127 & $14: 05: 22.82$ & $28: 30: 13.8$ & 14.88 & 13.56 & 12.21 & 122.8 & 6.7 \\
\hline 3342 & $14: 05: 23.30$ & $28: 29: 35.6$ & 16.74 & 16.67 & 16.51 & 122.0 & 5.7 \\
\hline $2891 *$ & $14: 05: 23.54$ & $28: 30: 41.7$ & 15.60 & 14.57 & 13.48 & 111.2 & 4.8 \\
\hline 1839 & $14: 05: 24.86$ & $28: 32: 11.1$ & 15.39 & 14.13 & 12.95 & 116.5 & 3.6 \\
\hline 1461 & $14: 05: 24.91$ & $28: 32: 40.5$ & 17.12 & 16.37 & 15.43 & 123.0 & 9.5 \\
\hline 1749 & $14: 05: 25.12$ & $28: 32: 18.0$ & 17.56 & 16.83 & 15.99 & 106.3 & 4.5 \\
\hline 3401 & $14: 05: 25.60$ & $28: 29: 22.9$ & 18.40 & 17.81 & 16.97 & 113.3 & 2.0 \\
\hline 3071 & $14: 05: 25.37$ & $28: 30: 23.1$ & 15.53 & 14.54 & 13.47 & 118.1 & 10.6 \\
\hline 3033 & $14: 05: 25.86$ & $28: 30: 27.4$ & 16.40 & 15.84 & 15.15 & 119.2 & 11.2 \\
\hline 2821 & $14: 05: 25.77$ & $28: 30: 50.1$ & 16.35 & 15.52 & 14.54 & 117.9 & 7.2 \\
\hline 2919 & $14: 05: 26.27$ & $28: 30: 40.4$ & 17.07 & 16.33 & 15.43 & 117.1 & 4.1 \\
\hline 2776 & $14: 05: 26.63$ & $28: 30: 55.2$ & 16.23 & 15.39 & 14.41 & 108.1 & 4.1 \\
\hline 1351 & $14: 05: 27.14$ & $28: 32: 51.2$ & 17.20 & 16.42 & 15.53 & 123.4 & 8.1 \\
\hline 1946 & $14: 05: 28.29$ & $28: 32: 04.3$ & 17.54 & 16.82 & 15.95 & 109.5 & 12.6 \\
\hline 2440 & $14: 05: 30.17$ & $28: 31: 25.0$ & 16.40 & 15.56 & 14.58 & 121.8 & 0.5 \\
\hline 2544 & $14: 05: 30.90$ & $28: 31: 16.1$ & 16.69 & 15.84 & 14.94 & 133.0 & 18.8 \\
\hline 1497 & $14: 05: 31.03$ & $28: 32: 39.9$ & 15.92 & 14.99 & 13.94 & 112.4 & 5.5 \\
\hline 2483 & $14: 05: 31.19$ & $28: 31: 21.8$ & 15.75 & 14.85 & 13.85 & 121.8 & 8.9 \\
\hline 1972 & $14: 05: 31.38$ & $28: 32: 02.8$ & 15.69 & 14.68 & 13.61 & 104.5 & 2.6 \\
\hline 1615 & $14: 05: 31.86$ & $28: 32: 30.7$ & 18.53 & 17.93 & 17.12 & 113.5 & 3.5 \\
\hline 826 & $14: 05: 32.65$ & $28: 33: 51.6$ & 17.40 & 16.69 & 15.85 & 122.2 & 16.1 \\
\hline 1534 & 14:05:33.09 & $28: 32: 37.8$ & 16.06 & 15.16 & 14.14 & 121.6 & 7.1 \\
\hline 904 & 14:05:33.09 & $28: 33: 41.7$ & 15.23 & 14.06 & 12.83 & 123.6 & 7.8 \\
\hline 707 & $14: 05: 33.53$ & $28: 34: 08.6$ & 16.71 & 15.91 & 14.98 & 130.2 & 8.5 \\
\hline $727 *$ & $14: 05: 34.44$ & 28:34:06.2 & 15.50 & 14.46 & 13.33 & 136.9 & 6.7 \\
\hline 1361 & $14: 05: 34.84$ & $28: 32: 52.5$ & 18.50 & 17.83 & 17.05 & 160.8 & 13.4 \\
\hline 1075 & $14: 05: 34.96$ & $28: 33: 20.6$ & 17.85 & 17.14 & 16.29 & 133.8 & 17.1 \\
\hline $1320^{*}$ & $14: 05: 35.22$ & $28: 32: 56.7$ & 15.62 & 14.61 & 13.51 & 131.5 & 6.3 \\
\hline 1024 & $14: 05: 35.55$ & $28: 33: 27.4$ & 18.20 & 17.57 & 16.74 & 129.3 & 5.1 \\
\hline 1112 & $14: 05: 36.00$ & $28: 33: 16.9$ & 18.33 & 17.65 & 16.86 & 112.4 & 3.9 \\
\hline 690 & $14: 05: 39.51$ & $28: 34: 14.4$ & 17.92 & 17.22 & 16.42 & 105.8 & 0.6 \\
\hline 2473 & $14: 05: 39.92$ & $28: 31: 25.2$ & 16.73 & 16.35 & 15.77 & 109.4 & 13.0 \\
\hline $2635^{*}$ & $14: 05: 44.53$ & $28: 31: 13.5$ & 15.56 & 14.62 & 13.55 & 103.0 & 5.6 \\
\hline 2629 & $14: 05: 39.28$ & $28: 31: 12.9$ & 14.89 & 13.61 & 12.27 & 108.1 & 7.0 \\
\hline 2642 & $14: 05: 42.19$ & $28: 31: 12.1$ & 16.76 & 16.71 & 16.63 & 111.0 & 13.0 \\
\hline $3176^{*}$ & $14: 05: 39.96$ & $28: 30: 11.1$ & 15.61 & 14.60 & 13.50 & 133.7 & 13.0 \\
\hline 3306 & $14: 05: 41.08$ & $28: 29: 48.2$ & 15.90 & 14.97 & 13.92 & 131.7 & 6.0 \\
\hline 837 & $14: 05: 39.30$ & $28: 33: 52.3$ & 17.13 & 16.36 & 15.46 & 121.2 & 6.7 \\
\hline 1398 & $14: 05: 29.10$ & $28: 32: 47.3$ & 15.32 & 14.48 & 13.48 & 123.2 & 7.4 \\
\hline 1344 & $14: 05: 26.71$ & $28: 32: 51.6$ & 18.66 & 18.20 & 17.34 & 103.1 & 41.7 \\
\hline 1106 & $14: 05: 32.58$ & $28: 33: 16.2$ & 18.89 & 18.40 & 17.63 & 75.8 & 17.1 \\
\hline 3387 & $14: 05: 20.09$ & $28: 29: 24.1$ & 19.21 & 18.66 & 17.88 & 132.8 & 6.9 \\
\hline 2725 & $14: 05: 22.37$ & $28: 30: 58.4$ & 18.82 & 18.23 & 17.41 & 123.8 & 29.8 \\
\hline 2957 & $14: 05: 22.72$ & $28: 30: 33.9$ & 18.09 & 17.64 & 16.82 & 94.9 & 9.4 \\
\hline 3192 & $14: 05: 22.77$ & 28:30:03.6 & 19.46 & 19.29 & 18.78 & 152.9 & 22.5 \\
\hline 3078 & $14: 05: 23.48$ & $28: 30: 21.4$ & 19.25 & 18.67 & 17.90 & 144.6 & 14.4 \\
\hline 946 & $14: 05: 33.66$ & $28: 33: 36.3$ & 19.03 & 18.46 & 17.71 & 108.8 & 15.2 \\
\hline 968 & $14: 05: 35.90$ & $28: 33: 33.7$ & 19.49 & 18.88 & 18.16 & 113.8 & 5.1 \\
\hline 888 & $14: 05: 36.45$ & $28: 33: 45.2$ & 18.64 & 18.08 & 17.28 & 115.3 & 8.1 \\
\hline 3113 & $14: 05: 26.33$ & $28: 30: 17.1$ & 18.96 & 18.35 & 17.58 & 122.3 & 16.3 \\
\hline \multicolumn{8}{|c|}{ Sample non-members } \\
\hline 1980 & $14: 05: 31.85$ & $28: 32: 02.6$ & 17.95 & 17.21 & 16.47 & 68.9 & 6.3 \\
\hline
\end{tabular}




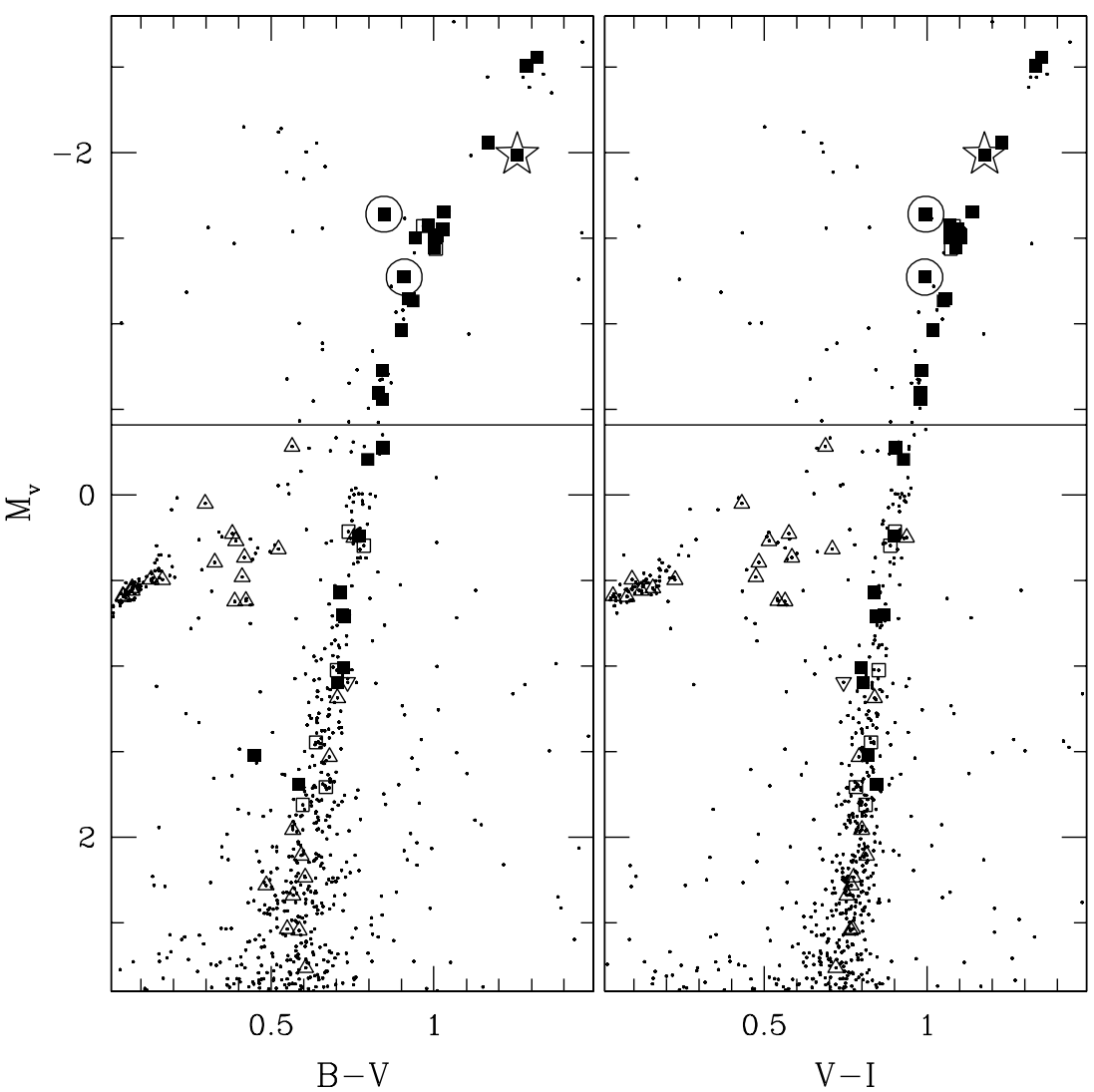

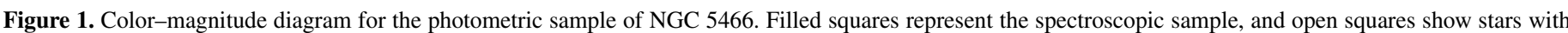

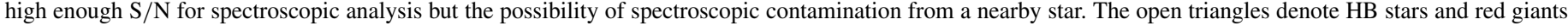

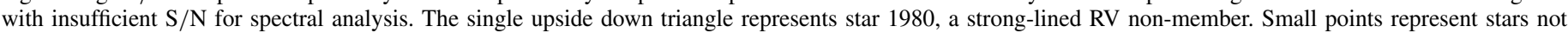

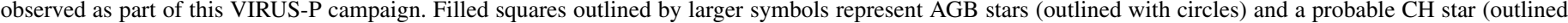
with a star).

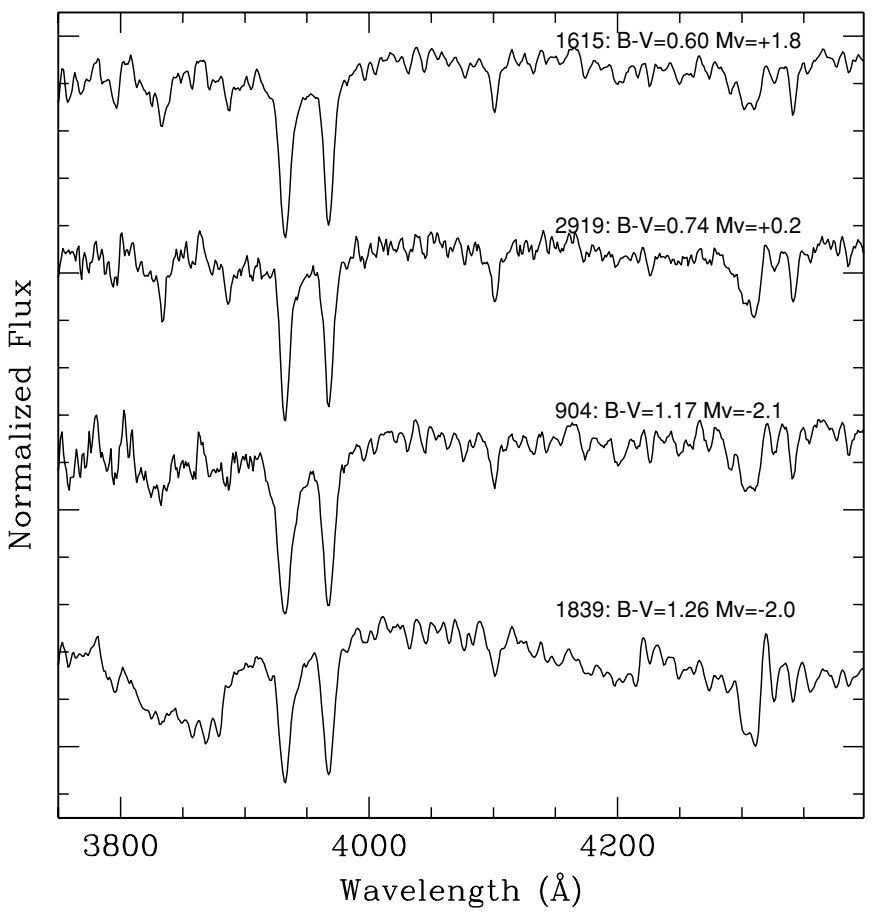

Figure 2. Four spectra from our spectroscopic sample as examples of the characteristics of VIRUS-P spectra. The top three spectra were chosen to span the range of absolute magnitude in our data set. The lowest spectrum, that of star 1839 , has very strong $\mathrm{CN}$ and $\mathrm{CH}$ bands. 1839 also sits to the red of the $\mathrm{RGB}$ in the $(V, B-V) \mathrm{CMD}$ and is probably a $\mathrm{CH}$ star. at the W. M. Keck Observatory on Mauna Kea. All light was directed to the blue arm of the spectrograph by the use of a mirror in place of a dichroic. The 400/3400 grism was used, resulting in a typical resolution of $7 \AA$ and a pixel spacing of $1 \AA$. Exposure times were between 420 and 600 s producing a mean $S / N$ of 225 pixel $^{-1}$ in the wavelength range $4000 \AA \leqslant \lambda \leqslant 4100 \AA$. All reductions from flat field division through flux calibration were carried out using the XIDL routines developed by J. X. Prochaska.

\section{RADIAL VELOCITY AND MEMBERSHIP}

For radial velocity determinations, data were cross-correlated with a template spectrum: for RGB and AGB stars, the template was the Hinkle Arcturus atlas (Hinkle et al. 2000) convolved with a Gaussian to create an $R=850$ spectrum. The HB star template was generated from a synthetic spectrum with $T_{\text {eff }}=6000 \mathrm{~K}, \log (g)=3.00 \mathrm{dex}$, and $[\mathrm{Fe} / \mathrm{H}]=-2.0 \mathrm{dex}$.

Figure 3 shows the distribution of our velocity measurements. The weighted average is $118.0 \pm 0.4 \mathrm{~km} \mathrm{~s}^{-1}$ with an $\mathrm{rms}$ of $17.0 \mathrm{~km} \mathrm{~s}^{-1}$. This rms is $5 \%$ of a resolution element with VIRUS-P. More customized template spectra may allow improvements in the rms scatter. The mean of literature values for the systemic velocity of NGC 5466 is $108 \mathrm{~km} \mathrm{~s}^{-1}$ (Harris 1996), and solid horizontal lines in Figure 4 show velocities from Pryor et al. (1991) and Peterson \& Latham (1986). The individual star radial velocities are listed in Table 1 . In the following analysis, spectra are individually shifted to rest wavelengths according to these radial velocities. 


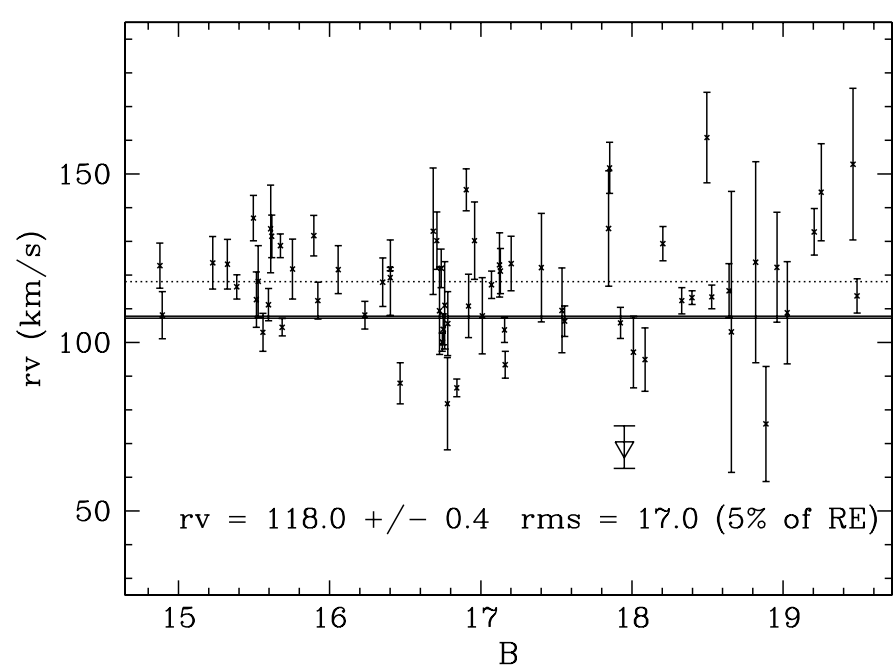

Figure 3. Radial velocities for all stars in our photometric sample, with a horizontal dashed line marking the mean velocity of $118.0 \mathrm{~km} \mathrm{~s}^{-1}$. The horizontal solid line (which is actually two separate lines that can be distinguished upon zooming in on the figure) represents the mean cluster radial velocity values taken from Peterson \& Latham (1986) and Pryor et al. (1991). The error on our mean velocity is $0.4 \mathrm{~km} \mathrm{~s}^{-1}$ and the rms is $17.0 \mathrm{~km} \mathrm{~s}^{-1}$. This $\mathrm{rms}$ is $5 \%$ of a resolution element with VIRUS-P. The single inverted triangle represents star 1980 , a strong-lined star that is also a radial velocity non-member.

\section{BAND STRENGTHS AND BIMODALITY}

$\mathrm{CN}$ and $\mathrm{CH}$ band strengths are typically quantified with indices that measure the magnitude difference between the integrated flux within the feature in question and the integrated flux in one or two nearby continuum regions, in the sense that more absorption in the feature produces a larger band-strength index. For $\mathrm{CN}$ band strength, we measure the index $S(3839)$, defined in Norris et al. (1981) as

$$
S(3839)=-2.5 \log \frac{\int_{3883}^{3916} I_{\lambda} d \lambda}{\int_{3846}^{3883} I_{\lambda} d \lambda} .
$$

The index we use for $\mathrm{CH}$ band strength is $S_{2}(\mathrm{CH})$, defined in Martell et al. (2008b) as

$$
S_{2}(\mathrm{CH})=-2.5 \log \frac{\int_{4297}^{4317} I_{\lambda} d \lambda}{\int_{4212}^{4242} I_{\lambda} d \lambda+\int_{4330}^{4375} I_{\lambda} d \lambda} .
$$

Band-strength indices tend to be designed to be effective for a single stellar population, for example, $\mathrm{CH}(4300)$ (Harbeck et al. 2003, designed for main-sequence stars in 47 Tucanae) and $\mathrm{CH}(G)$ (Lee 1999, designed for bright red giants in M3), and can be difficult to apply outside the parameter space for which they were intended. $S_{2}(\mathrm{CH})$ was specifically designed to be sensitive to variations in carbon abundance in bright red giants $\left(M_{V} \leqslant-1.0\right)$ across a wide range of metallicity, and therefore ought to be valid for the sample at hand.

The errors on our band strengths have two components, errors related to the fluxing and errors related to the $\mathrm{S} / \mathrm{N}$. S/N errors were calculated by propagating the Poisson noise errors through the formulas for the band indices. To estimate the errors in our fluxing, we tried several techniques to reflux the data including using synthetic spectra matched to each star's photometric temperature and using average flux distributions for color bins along the giant branch. The two sources of error were added in quadrature, and the total errors are reported in Table 2 as $\sigma_{S}$ and $\sigma_{S 2}$ for $S(3839)$ and $S_{2}(\mathrm{CH})$, respectively. For the March observing run the $\mathrm{S} / \mathrm{N}$ in the $S_{2}(\mathrm{CH})$ band was quite high and the fluxing errors dominate while for the July observing run, where only a single dither set was taken for the final fourth field, the Poisson noise contribution plays a significant contribution to the total error. For $S(3839)$, the Poisson error tends to dominate the total error in all cases. Errors in radial velocity are typically $17 \mathrm{~km} \mathrm{~s}^{-1}$, or $5 \%$ of a pixel, as mentioned in Section 3, and do not have a significant effect on measured band strengths. Shifting our wavelengths to the blue or red by $17 \mathrm{~km} \mathrm{~s}^{-1}$ changes the measured $S_{2}(\mathrm{CH})$ by $0.001 \mathrm{mag}$, which is small relative to the fluxing and noise-related errors in $S_{2}(\mathrm{CH})$.

Five of the stars in the LRIS data set are also in the VIRUS-P spectroscopic sample, and the $\mathrm{CN}$ and $\mathrm{CH}$ band strengths measured from the LRIS spectra are quite similar

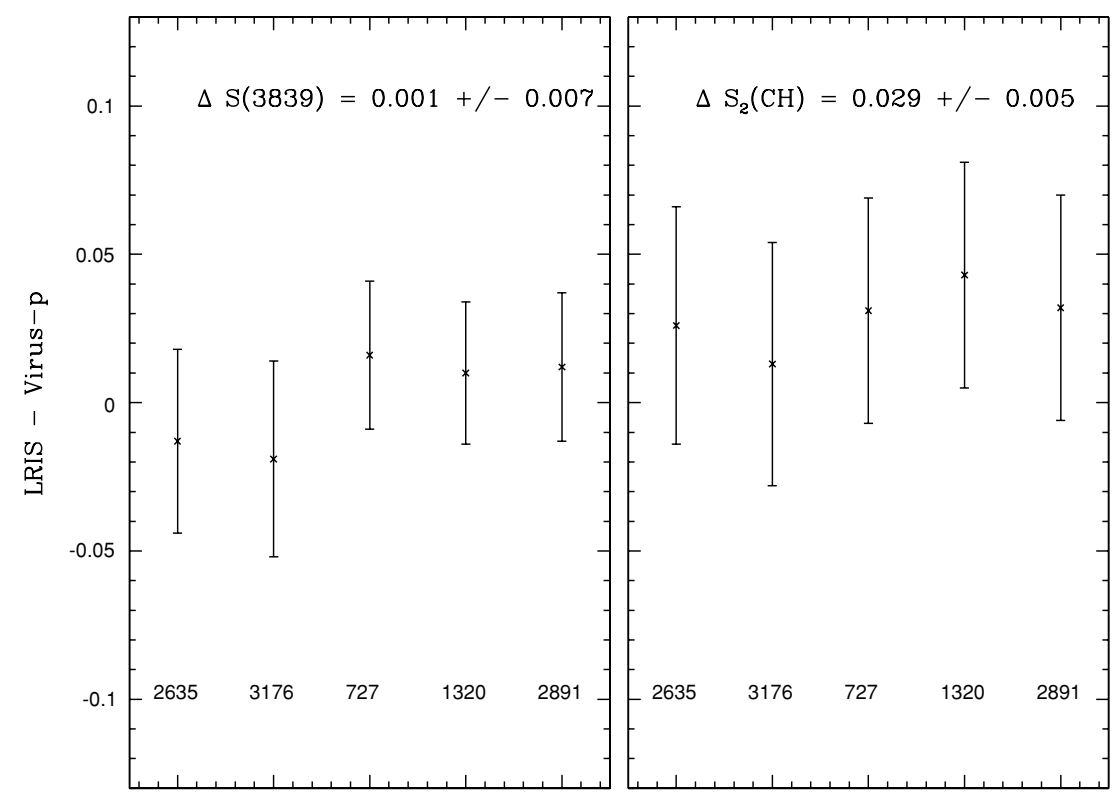

Figure 4. Comparison of band indices measured from the Keck LRIS spectra discussed in Section 2.1 and those measured from VIRUS-P spectra for the five stars in common to both data sets. Identification numbers are given at the bottom of each panel. 
Table 2

Results

\begin{tabular}{|c|c|c|c|c|c|c|}
\hline ID & $S(3839)$ & $\sigma_{S}$ & $S_{2}(\mathrm{CH})$ & $\sigma_{S 2}$ & {$[\mathrm{C} / \mathrm{Fe}]$} & $\overline{\sigma_{[\mathrm{C} / \mathrm{Fe}]}}$ \\
\hline \multicolumn{7}{|c|}{ Main sample } \\
\hline 1839 & 0.460 & 0.027 & 0.793 & 0.038 & 0.02 & 0.18 \\
\hline 1398 & -0.185 & 0.034 & 0.603 & 0.042 & -0.68 & 0.18 \\
\hline 2483 & -0.199 & 0.026 & 0.609 & 0.039 & -0.81 & 0.14 \\
\hline 2629 & -0.093 & 0.055 & 0.510 & 0.046 & -1.14 & 0.26 \\
\hline 3176 & -0.028 & 0.033 & 0.622 & 0.041 & -0.69 & 0.13 \\
\hline 3306 & -0.160 & 0.031 & 0.663 & 0.040 & -0.60 & 0.43 \\
\hline 2635 & -0.144 & 0.031 & 0.596 & 0.040 & -0.87 & 0.17 \\
\hline 3127 & -0.040 & 0.024 & 0.522 & 0.037 & -0.91 & 0.13 \\
\hline 904 & -0.087 & 0.023 & 0.591 & 0.038 & -0.80 & 0.18 \\
\hline 727 & -0.135 & 0.025 & 0.648 & 0.038 & -0.49 & 0.14 \\
\hline 3071 & -0.166 & 0.037 & 0.587 & 0.042 & -0.86 & 0.13 \\
\hline 2891 & -0.110 & 0.025 & 0.636 & 0.038 & -0.58 & 0.11 \\
\hline 1320 & -0.139 & 0.024 & 0.630 & 0.038 & -0.58 & 0.11 \\
\hline 3305 & -0.144 & 0.026 & 0.658 & 0.038 & -0.48 & 0.12 \\
\hline 1497 & -0.102 & 0.026 & 0.645 & 0.038 & -0.52 & 0.11 \\
\hline 1534 & -0.166 & 0.024 & 0.648 & 0.038 & -0.47 & 0.11 \\
\hline 2776 & -0.145 & 0.026 & 0.664 & 0.039 & -0.39 & 0.11 \\
\hline 2821 & -0.209 & 0.029 & 0.688 & 0.040 & -0.25 & 0.12 \\
\hline 2440 & -0.116 & 0.029 & 0.638 & 0.040 & -0.52 & 0.11 \\
\hline 2544 & -0.218 & 0.028 & 0.656 & 0.040 & -0.42 & 0.13 \\
\hline 707 & -0.206 & 0.026 & 0.709 & 0.039 & -0.08 & 0.12 \\
\hline 1749 & -0.168 & 0.039 & 0.673 & 0.045 & -0.11 & 0.13 \\
\hline 826 & -0.138 & 0.034 & 0.685 & 0.042 & -0.05 & 0.10 \\
\hline 1946 & -0.185 & 0.037 & 0.701 & 0.044 & -0.05 & 0.11 \\
\hline 3401 & -0.203 & 0.032 & 0.685 & 0.041 & -0.01 & 0.14 \\
\hline 690 & -0.265 & 0.054 & 0.736 & 0.054 & 0.16 & 0.15 \\
\hline 2753 & -0.239 & 0.044 & 0.709 & 0.048 & -0.05 & 0.21 \\
\hline 837 & -0.214 & 0.031 & 0.710 & 0.041 & 0.02 & 0.13 \\
\hline 2957 & -0.238 & 0.091 & 0.660 & 0.079 & 0.12 & 0.12 \\
\hline \multicolumn{7}{|c|}{ Sample with potential blends } \\
\hline 2919 & -0.209 & 0.042 & 0.726 & 0.046 & 0.01 & 0.12 \\
\hline 1351 & -0.188 & 0.031 & 0.712 & 0.041 & -0.05 & 0.14 \\
\hline 1075 & -0.220 & 0.036 & 0.704 & 0.044 & 0.05 & 0.11 \\
\hline 1361 & -0.184 & 0.053 & 0.663 & 0.053 & -0.03 & 0.13 \\
\hline 1615 & -0.215 & 0.036 & 0.685 & 0.043 & 0.06 & 0.10 \\
\hline 1024 & -0.191 & 0.038 & 0.659 & 0.045 & -0.12 & 0.15 \\
\hline 2481 & -0.174 & 0.039 & 0.560 & 0.043 & -0.96 & 0.11 \\
\hline 1972 & -0.088 & 0.066 & 0.631 & 0.055 & -0.56 & 0.11 \\
\hline
\end{tabular}

to band strengths measured from the VIRUS-P data. Figure 4 shows $\Delta S(3839)$ and $\Delta S_{2}(\mathrm{CH})$ for the stars in common between the two data sets, in the sense LRIS-VIRUS-P, and differences between the two data sets are quite small. The band strengths given in Table 2 come exclusively from the VIRUS-P data.

Since the $3883 \AA \mathrm{CN}$ band strength can be used as a proxy for nitrogen abundance, and the $4320 \AA \mathrm{CH}$ band strength for carbon abundance, a comparison of $\mathrm{CN}$ and $\mathrm{CH}$ band strength in a globular cluster ought to reveal anticorrelated abundance behavior. As can be seen in Figure 5, the CN band strength in our sample rises slightly with rising luminosity, but does not show a large range at fixed $M_{V}$. The clear outlier, with a large open star drawn around it, is star 1839 , the star with the unusually strong $\mathrm{CN}$ and $\mathrm{CH}$ features in Figure 2. It is excluded from the rest of our band strength and abundance analysis.

The $S(3839)$ band strengths are correlated, to first order, with luminosity. To better distinguish stars that are $\mathrm{CN}$-weak from stars that are $\mathrm{CN}$-strong, we fit a linear relationship between $S(3839)$ and $M_{V}$ and evaluated each star based on its vertical distance from this linear relationship $(\delta S(3839))$. We divide the sample into relatively $\mathrm{CN}$-weak (negative $\delta S(3839)$, open

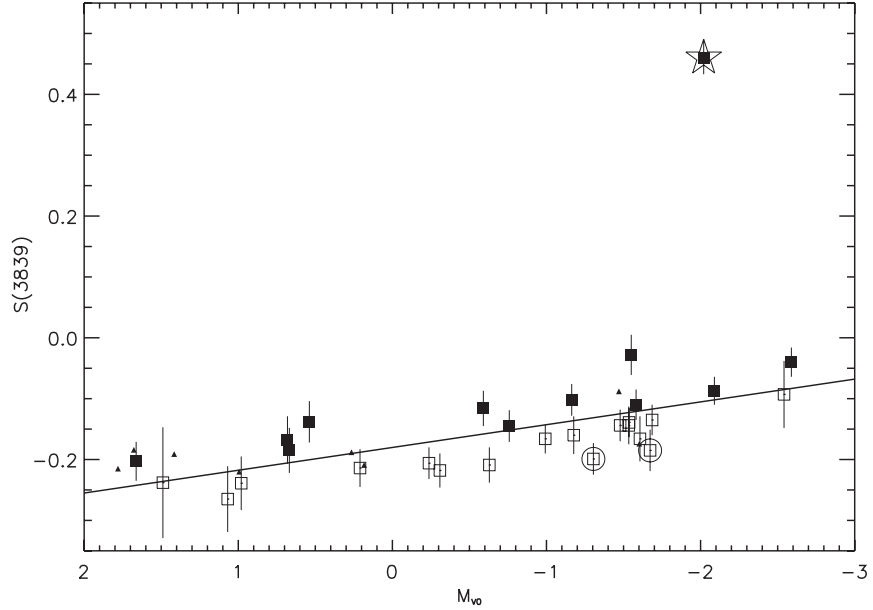

Figure 5. CN band-strength index $S(3839)$ vs. absolute $V$ magnitude for all stars in our spectroscopic sample. The solid line is a least-squares fit, and we classify stars that lie above the line (shown as filled squares) as relatively $\mathrm{CN}$-strong and stars that lie below the line (drawn as open squares) as relatively $\mathrm{CN}$-weak. Small triangles denote stars with sufficient $\mathrm{S} / \mathrm{N}$ for spectroscopic analysis but possible spectroscopic blending. The clear outlier, outlined with a large open star, is star 1839, noted in Figure 2 as a probable $\mathrm{CH}$ star. The two points outlined with large open circles (stars 1398 and 2483) lie to the blue of the RGB in Figure 1, and are likely AGB stars.

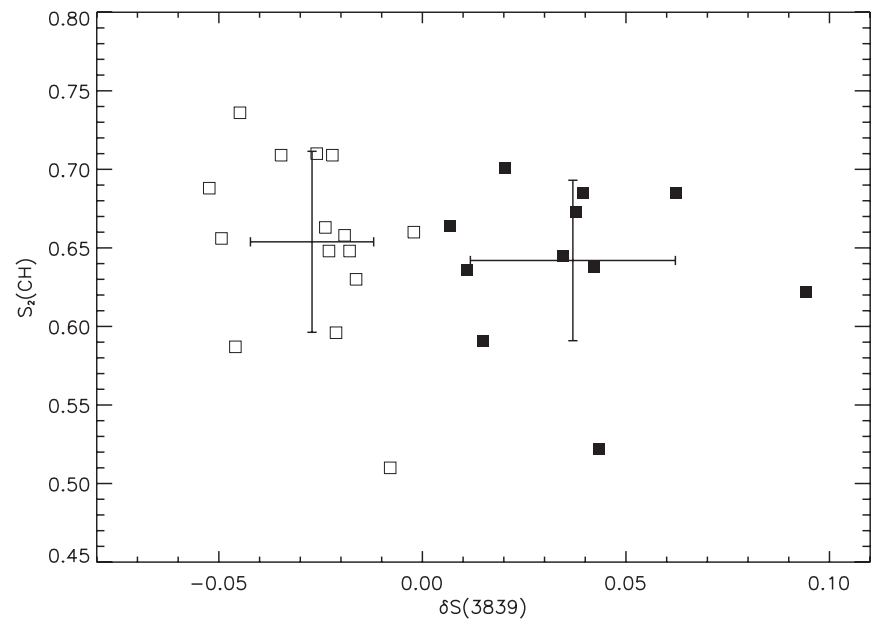

Figure 6. $\mathrm{CN}$ band-strength index $\delta S(3839)$ vs. $\mathrm{CH}$ band-strength index $S_{2}(\mathrm{CH})$ for the spectroscopic sample. The symbols are the same as in Figure 5. The large crosses are centered at the mean values of $\delta S(3839)$ and $S_{2}(\mathrm{CH})$ for the $\mathrm{CN}$-weak and $\mathrm{CN}$-strong groups, and their sizes are the standard deviations of the indices. $\mathrm{CN}$ and $\mathrm{CH}$ band strengths are typically anticorrelated in globular cluster stars, indicating anticorrelated carbon and nitrogen abundances. The lack of strong anticorrelation here is the result of a smaller than typical range in $\mathrm{CN}$ band strength in NGC 5466, and possibly a smaller range in $[\mathrm{C} / \mathrm{Fe}]$ and $[\mathrm{N} / \mathrm{Fe}]$ than in higher-metallicity globular clusters.

squares) and $\mathrm{CN}$-strong (positive $\delta S(3839)$, solid squares) stars. Unlike in studies of higher-metallicity globular clusters (see Norris et al. 1981 for a typical example), there is not a clear gap between the relatively $\mathrm{CN}$-weak and $\mathrm{CN}$-strong groups. In Figure 6, we compare $\delta S(3839)$ and $S_{2}(\mathrm{CH})$ band strengths, with the same symbols (open/solid squares) representing $\mathrm{CN}$ class, and there is not a strong anticorrelation between $\mathrm{CN}$ class and $\mathrm{CH}$ band strength. The two large crosses are centered at the mean values for $\delta S(3839)$ and $S_{2}(\mathrm{CH})$ for the two groups, with their size set to equal the standard deviations. They are not obviously offset from each other in $S_{2}(\mathrm{CH})$. This may be simply an effect of the extremely limited range in $\mathrm{CN}$ and $\mathrm{CH}$ band strengths permitted by the low overall metallicity of NGC 5466 . 


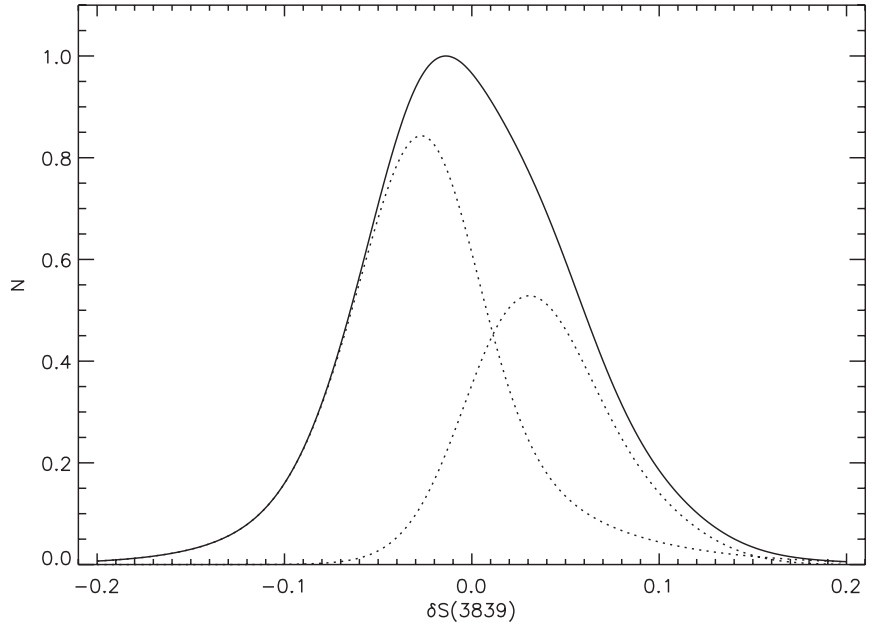

Figure 7. Solid curve is a generalized histogram for the luminosity-independent $\mathrm{CN}$ band-strength measure $\delta S(3839)$ measured for our full spectroscopic sample, and the dashed curves are generalized histograms for $\delta S(3839)$ in the relatively $\mathrm{CN}$-weak and $\mathrm{CN}$-strong groups calculated independently.

Another way to visualize the distribution of $\mathrm{CN}$ band strength in our data set is the generalized histogram, which is constructed by representing each star as a Gaussian in $\delta S(3839)$ with a width equal to the measurement error $\sigma_{S}$, and then summing the individual Gaussians. The result is shown in Figure 7, in which the solid curve is the generalized histogram for the full data set and the dashed curves are the generalized histograms calculated just for the relatively $\mathrm{CN}$-weak and $\mathrm{CN}$-strong groups. The upper panel of Figure 8 shows the single Gaussian that best fits the generalized histogram from Figure 7 , and the lower panel, showing the residual between the best-fit curve and the data, shows a clearly double-peaked shape. This double-peaked residual suggests the system is better fit by two Gaussians.

Based on our analysis of the generalized histogram, we claim that NGC 5466 contains two closely spaced groups in CN band strength, a result of intrinsic ranges in carbon and nitrogen abundance and low overall metallicity. The lack of a gap in $\mathrm{CN}$ band strength between the two groups of stars in Figure 5 is seen here as a fairly small distance between the peaks of the $\mathrm{CN}$-weak and $\mathrm{CN}$-strong generalized histograms. Similarly, metal-poor globular clusters such as M15 (Lee 2000) and M53 (Martell et al. 2008a) also exhibit small separations between their $\mathrm{CN}$-weak and $\mathrm{CN}$-strong groups and a small overall range in $S$ (3839) (among red giants in the three clusters $\delta S(3839)$ has a standard deviation of 0.038 in NGC 5466, 0.056 in M53, and 0.037 in M15). This suggests that the range of carbon and nitrogen abundance in low-metallicity globular clusters may be smaller than is typical for more moderate-metallicity clusters. In addition, since the measurement errors on $S(3839)$ control the widths of the individual Gaussians comprising the generalized histogram, they can have strong effects on our interpretation of it, with very large measurement errors potentially rendering two closely spaced populations indistinguishable from a single broad distribution.

To understand the effects of measurement error on our generalized histogram, we performed a Monte Carlo test exploring how the size of our measurement errors affects the Kolmogorov-Smirnov (K-S) coefficient $p$, which quantifies the probability that the $\mathrm{CN}$-strong and $\mathrm{CN}$-weak groups were drawn from the same initial population. We rescaled our true measurement errors $\sigma_{S}$ by factors ranging from 0.5 to 10 , then selected a new value for each data point from within a Gaussian distribu-

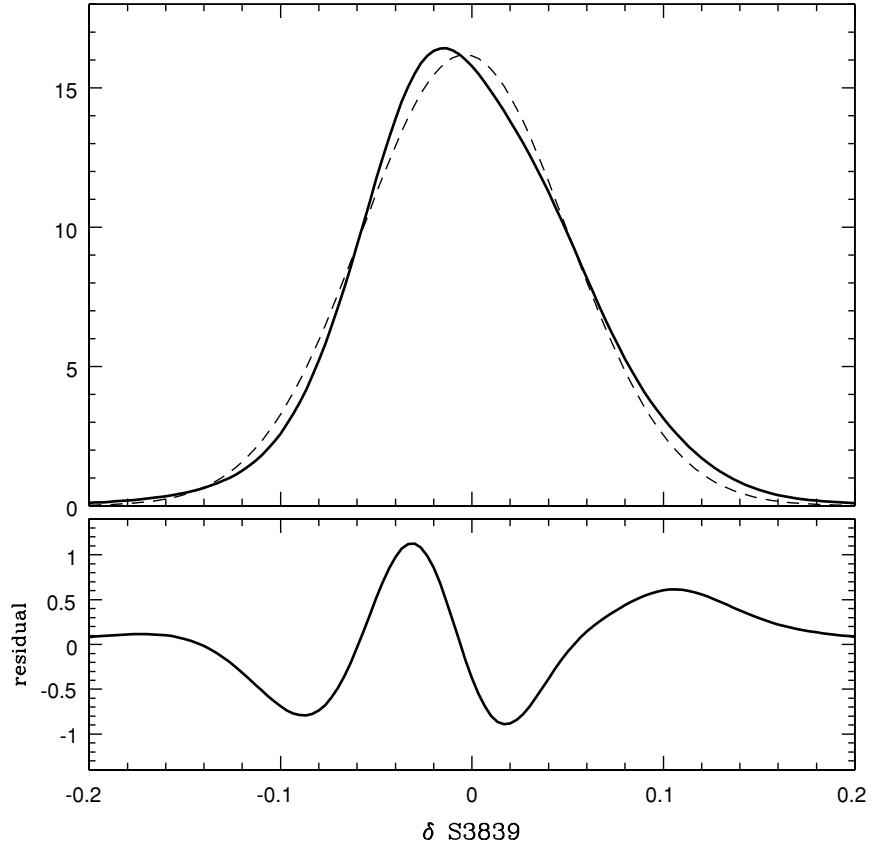

Figure 8. Upper panel shows the generalized histogram from Figure 7 as a solid line, with a simple Gaussian fit to the distribution overplotted as a dotted line. The lower panel shows the difference between the two curves, and there is a clear two-peaked shape, which suggests that the data are best represented as two overlapping Gaussian distributions.

tion centered at the measured value of $\delta S(3839)$, with a width of the rescaled measurement error. This reselection was done for the $\mathrm{CN}$-normal and $\mathrm{CN}$-strong groups identified in Figure 4 independently. The K-S $p$ coefficient was then measured as an indicator of whether the two $\mathrm{CN}$ band-strength groups containing reselected points with rescaled errors seemed likely to have been drawn from the same initial population. Reselections were done $10^{4}$ times for each rescaling of the measurement error, and we find that $p$ is strongly concentrated near zero when the rescaling factor is relatively small: for a rescaling factor of 1 , $99 \%$ of realizations have a $p$ less than 0.1 and none have a $p$ larger than 0.61 , and $82 \%$ of realizations with a rescaling factor of 3 have a $p$ less than 0.5 . With a rescaling factor of 10 , we find that $p$ values are fairly evenly distributed between 0 and 1 , with $51 \%$ below $p=0.5$. Based on this test, we are fairly confident that our two $\mathrm{CN}$ groups really are distinct, even if our errors in $S(3839)$ are underestimated by a factor of 3 .

\section{EVOLUTION OF CARBON ABUNDANCE}

Carbon abundances were determined by spectral synthesis of the $G$-band region. For each star, a set of synthetic spectra were created with a single $T_{\text {eff }}, \log (g)$, and $[\mathrm{Fe} / \mathrm{H}]$, and a range of possible values for $[\mathrm{C} / \mathrm{Fe}]$, smoothed to the resolution of the VIRUS-P data. The synthetic spectra were generated using a modified version of MOOG 2009 (Sneden 1973) which generates fluxed spectra instead of normalized spectra. We used the PLEZ2000 grid of metal-poor model atmospheres (Plez 2000). The temperatures and surface gravities were derived from the photometry in Table 1 using the prescriptions in Ramirez \& Melendez (2005). We adopted $[\alpha / \mathrm{Fe}]=0.3,{ }^{12} \mathrm{C} /{ }^{13} \mathrm{C}=6$, and $[\mathrm{Fe} / \mathrm{H}]=-2.0$ for all stars. The choice of the carbon isotope ratio does make a small difference to the derived total carbon abundance. At these metallicities, where the lines are reasonably weak, a change of ${ }^{12} \mathrm{C} /{ }^{13} \mathrm{C}$ from 6 to 50 would make about 0.06 dex difference to $[\mathrm{C} / \mathrm{Fe}]$ which is much smaller than 


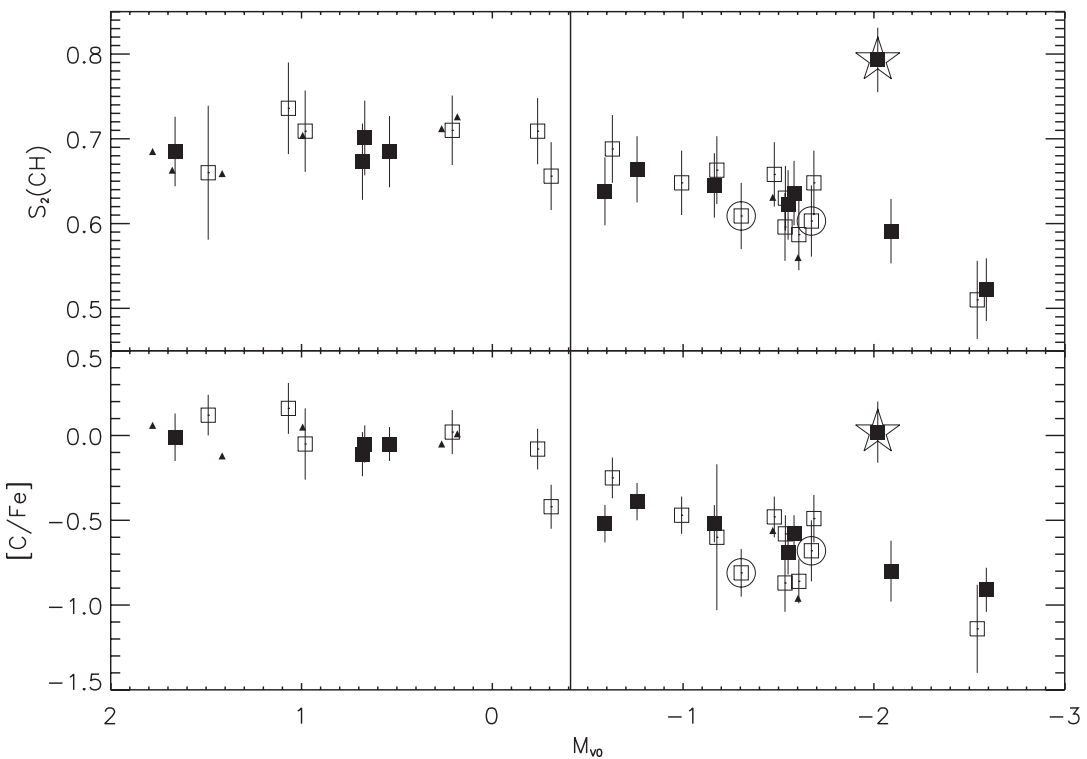

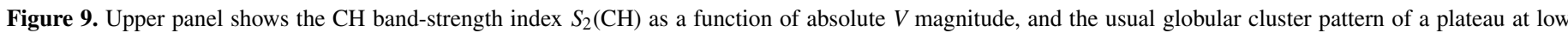

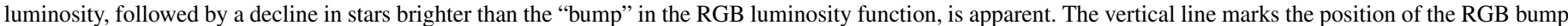

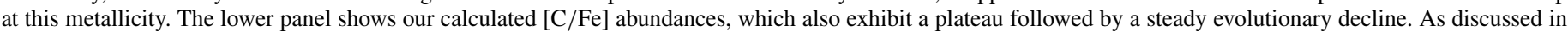
the text, the rate of carbon depletion measured in NGC 5466 is consistent with the low-metallicity carbon depletion rate found in Martell et al. (2008c).

the quoted errors. The synthetic spectrum yielding the residual (in the sense observed-synthetic) that is closest to zero in the region of the $\mathrm{CH} G$ band was chosen to have the correct carbon abundance. Table 2 lists ID numbers, VIRUS-P CN and $\mathrm{CH}$ band strengths, and carbon abundances along with their associated errors for the stars in the spectroscopic sample and for those that have good $\mathrm{S} / \mathrm{N}$ but are possibly spectroscopically blended.

Observational studies of $\mathrm{CH}$ band strengths and $[\mathrm{C} / \mathrm{Fe}]$ abundances in red giants in globular clusters and in the field (e.g., Gratton et al. 2000; Smith \& Martell 2003; Smith et al. 2005) have clearly shown that bright, low-mass red giants in all Galactic environments experience steady carbon depletion as they evolve along the giant branch. This depletion only occurs in stars brighter than the "bump" in the RGB luminosity function, and proceeds at a rate that is metallicity dependent. The homogeneity and wide luminosity range of our spectroscopic sample makes it very useful for careful study of the rate and point of onset of deep mixing in NGC 5466.

The first dredge-up process that occurs when a star moves on to the RGB (Iben 1965, 1968) is caused by the base of the convective envelope dropping in to small radius, until hydrogen shell burning ignites, equilibrates, and restores the stability against convection of much of the stellar interior, driving the base of the convective zone to larger radius. This event homogenizes all material in the star outside the minimum radius reached by the convective envelope, and creates a discontinuity in the mean molecular weight $\mu$ at that radius. This $\mu$ discontinuity is destroyed when the hydrogen-burning shell, constantly moving outward in radius in search of fuel, encounters it. When that happens, the sudden change in the composition of the fuel available to the hydrogen-burning shell causes a brief loop in the star's path through the color-magnitude diagram: it becomes hotter and dimmer before recovering its equilibrium and continuing to evolve along the RGB. This evolutionary stutter causes a pile-up of stars in the luminosity function of the RGB, known as the "RGB bump" (see, e.g., Fusi Pecci et al. 1990). The time at which a red giant's hydrogenburning shell crosses the $\mu$ discontinuity is a unique function of stellar mass and metallicity, meaning that the absolute $V$ magnitude of the RGB bump can be calculated empirically or theoretically for a given globular cluster.

This destruction of the $\mu$ barrier permits slow mixing between the surface and the hydrogen shell, which results in a continuous decline in surface carbon abundance and an associated decline in $\mathrm{CH}$ band strength starting at the RGB bump and continuing for as long as a star is on the giant branch (see, e.g., Palacios et al. 2006; Denissenkov \& VandenBerg 2003). The structure of the hydrogen-burning shell is also a function of stellar metallicity, and is more compressed in higher-metallicity stars (see, e.g., Sweigart \& Mengel 1979 for a discussion of hydrogen-burning shell structure). The more radially extended structure of the hydrogen-burning shell in lower-metallicity stars allows deep mixing currents to carry material more efficiently from the stellar surface into the zone in which the $\mathrm{CN}$ cycle is operating, and return carbon-depleted, nitrogen-enhanced material to the surface. The rate of carbon depletion at the surface, then, carries a fair amount of information about the internal structure of the star, and also about the process that drives deep mixing currents (e.g., Eggleton et al. 2008).

The upper panel of Figure 9 shows $\mathrm{CH}$ band strength as a function of absolute $V$ magnitude, and the expected pattern of a plateau followed by a steady decline with rising luminosity can be seen. We calculate that the RGB bump ought to be at $M_{V}=-0.41$, using the relation $M_{V}^{\text {bump }}=0.94[\mathrm{Fe} / \mathrm{H}]+1.5$ found empirically in Fusi Pecci et al. (1990), and the vertical line in Figure 9 is at that luminosity. It is unclear whether our data completely agree with this prediction; given the sparseness of the data in the range $-0.5 \leqslant M_{V} \leqslant 0.0$, the corner between the plateau and the evolutionary decline could be anywhere within that range.

The lower panel of Figure 9 shows our derived carbon abundances as a function of absolute $V$ magnitude, and $[\mathrm{C} / \mathrm{Fe}]$ shows the same plateau, corner, and decline above the luminosity of the RGB bump $\left(M_{V} \simeq-0.4\right)$ as $S_{2}(\mathrm{CH})$ does. The carbon abundance before the onset of deep mixing is roughly solar, and drops by a dex by the tip of the giant branch. As was demonstrated in Martell et al. (2008c), carbon depletion in 
globular cluster red giants proceeds roughly twice as fast in the low-metallicity clusters M92 and M15 as it does in the highermetallicity clusters M5 and NGC 6712. Using Yale-Yonsei evolutionary tracks (Demarque et al. 2004), we determine the age difference between a star at $M_{V}=-0.41$ (the height of the RGB bump) and $M_{V}=-1.5$ to be $20 \mathrm{Myr}$, implying a carbon depletion rate of roughly $34 \mathrm{dex} \mathrm{Gyr}^{-1}$. This is in good agreement with the result of Martell et al. (2008c), which found a carbon depletion rate of 20-30 dex $\mathrm{Gyr}^{-1}$ for globular clusters with metallicities between -2.0 and -2.5 .

\section{SUMMARY}

We do not see strong star-to-star variations in $\mathrm{CN}$ or $\mathrm{CH}$ band strength in NGC 5466, but we do find that a sum of two Gaussians represents the generalized histogram of $\mathrm{CN}$ band strength better than a single Gaussian curve. This suggests that the underlying $S(3839)$ distribution is likely to consist of two groups that are distinct but not widely separated. There is a very weak anticorrelation between $\mathrm{CN}$ and $\mathrm{CH}$ band indices, but it is muted even compared to similarly metal-poor clusters. It may be that NGC 5466 experienced weaker primordial enrichment than other Galactic globular clusters, and even other low-metallicity globular clusters. It would be interesting to investigate whether theoretical scenarios for primordial enrichment of globular clusters predict varying enrichment efficiencies for particularly low-metallicity or low-mass clusters.

Although the initial abundances in NGC 5466 may exhibit less of a range than abundances in typical globular clusters, the stellar evolution-driven abundance changes match well with what is expected from the literature. Surface carbon depletion, a result of slow circulation of material between the photosphere and the hydrogen-burning shell in bright red giants, begins near the "bump" in the RGB luminosity function and proceeds at a rate consistent with the observations of Martell et al. (2008c) and the predictions of Denissenkov \& VandenBerg (2003).

The data set presented here is particularly well suited to the study of deep mixing in the red giants in NGC 5466, since the data extends to quite faint magnitudes. This is made possible by the multiplexing ability of VIRUS-P as an integral field spectrograph. We also show that VIRUS-P can be used to get velocities for stars to better than $17 \mathrm{~km} \mathrm{~s}^{-1}$ if good template matching is done. The future VIRUS instrument on the Hobby-Eberly Telescope will have comparable spectral resolution. However, instead of a single spectrograph it will have more than $100 \mathrm{IFU}$ spectrographs, and the fiber size on the sky will shrink from 4".1 to 1 .5. This will make VIRUS a very capable instrument for observing larger, more crowded globular clusters.

We thank the Cynthia and George Mitchell Foundation for funding the VIRUS-P instrument. Support for R.W. was provided by the National Science Foundation through AST0649128, which funds the McDonald Observatory REU program.

Support for S.L.M. and G.H.S. was provided by the NSF grant AST-0406988.

The authors recognize and acknowledge the very significant cultural role and reverence that the summit of Mauna Kea has always had within the indigenous Hawaiian community. We are most fortunate to have the opportunity to conduct observations from this mountain.

\section{REFERENCES}

Bellman, S., Briley, M. M., Smith, G. H., \& Claver, C. F. 2001, PASP, 113, 326 Bond, H. E. 2005, AJ, 129, 2914

Bond, H. E., \& Neff, J. S. 1969, ApJ, 158, 1235

Briley, M. M., Cohen, J. G., \& Stetson, P. B. 2004, AJ, 127, 1579

Briley, M. M., Smith, G. H., Hesser, J. E., \& Bell, R. A. 1993, AJ, 106, 142

Carbon, D. F., Romanishin, W., Langer, G. E., Butler, D., Kemper, E., Trefzger, C. F., Kraft, R. P., \& Suntzeff, N. B. 1982, ApJS, 49, 207

Carretta, E., et al. 2009, A\&A, 505, 117

Cohen, J. G., Briley, M. M., \& Stetson, P. B. 2005, AJ, 130, 1177

Demarque, P., Woo, J., Kim, Y., \& Yi, S. K. 2004, ApJS, 155, 667

Denissenkov, P. A., \& VandenBerg, D. A. 2003, ApJ, 593, 509

D'Ercole, A., Vesperini, E., D'Antona, F., McMillan, S. L. W., \& Recchi, S. 2008, MNRAS, 391, 825

Dierckx, P. 1993, Curve and Surface Fitting with Splines Monographs on Numerical Analysis (Oxford: Clarendon)

Dotter, A., et al. 2010, ApJ, 708, 698

Eggleton, P. P., Dearborn, D. S. P., \& Lattanzio, J. C. 2008, ApJ, 677, 581

Fusi Pecci, F., Ferraro, F. R., Crocker, D. A., Rood, R. T., \& Buonanno, R. 1990, A\&A, 238, 95

Gratton, R., Sneden, C., \& Carretta, E. 2004, ARA\&A, 42, 385

Gratton, R. G., Sneden, C., Carretta, E., \& Bragaglia, A. 2000, A\&A, 354, 169

Gratton, R. G., et al. 2001, A\&A, 369, 87

Harbeck, D., Smith, G. H., \& Grebel, E. K. 2003, AJ, 125, 197

Harris, W. E. 1996, AJ, 112, 1487

Hill, G. J., et al. 2008, Proc. SPIE, 7014, 701470

Hinkle, K., Wallace, L., Valenti, J., \& Harmer, D. (ed.) 2000, in Visible and Near Infrared Atlas of the Arcturus Spectrum 3727-9300 Å, (San Francisco, CA: ASP)

Iben, I., Jr. 1965, ApJ, 142, 1447

Iben, I., Jr. 1968, ApJ, 154, 581

Kelson, D. D. 2003, PASP, 115, 688

Kraft, R. P. 1994, PASP, 106, 553

Landolt, A. U. 1992, AJ, 104, 340

Langer, G. E., Kraft, R. P., Carbon, D. F., Friel, E., \& Oke, J. B. 1986, PASP, 98,473

Langer, G. E., Suntzeff, N. B., \& Kraft, R. P. 1992, PASP, 104, 523

Lee, S. G. 1999, AJ, 118, 920

Lee, S. G. 2000, J. Korean Astron. Soc., 33, 137

Martell, S. L., Smith, G. H., \& Briley, M. M. 2008a, PASP, 120, 7

Martell, S. L., Smith, G. H., \& Briley, M. M. 2008b, PASP, 120, 839

Martell, S. L., Smith, G. H., \& Briley, M. M. 2008c, AJ, 136, 2522

Norris, J., Cottrell, P. L., Freeman, K. C., \& Da Costa, G. S. 1981, ApJ, 244 205

Norris, J., \& Freeman, K. C. 1979, ApJ, 230, L179

Norris, J., \& Pilachowski, C. A. 1985, ApJ, 299, 295

Oke, J. B., et al. 1995, PASP, 107, 375

Palacios, A., Charbonnel, C., Talon, S., \& Siess, L. 2006, A\&A, 453, 261

Peterson, R. C., \& Latham, D. W. 1986, ApJ, 305, 645

Piotto, G. 2008, Mem. Soc. Astron. Ital., 79, 334

Plez, B. 2000, in IAU Symp. 177, The Carbon Star Phenomenon, ed. R. F. Wing (Cambridge: Cambridge Univ. Press), 71

Pryor, C., McClure, R. D., Fletcher, J. M., \& Hesser, J. E. 1991, AJ, 102, 1026

Ramirez, I., \& Melendez, J. 2005, ApJ, 626, 465

Shetrone, M. D. 1996, AJ, 112, 2639

Siegel, M. H., \& Bond, H. E. 2005, AJ, 129, 2924

Siegel, M. H., Majewski, S. R., Reid, I. N., \& Thompson, I. B. 2002, ApJ, 578, 151

Smith, G. H., Briley, M. M., \& Harbeck, D. 2005, AJ, 129, 1589

Smith, G. H., \& Martell, S. L. 2003, PASP, 115, 1211

Sneden, C. 1973, ApJ, 184, 839

Sneden, C., Kraft, R. P., Prosser, C. F., \& Langer, G. E. 1991, AJ, 102, 2001

Sneden, C., Kraft, R. P., Shetrone, M. D., Smith, G. H., Langer, G. E., \& Prosser, C. F. 1997, AJ, 114, 1964

Stetson, P. B. 1987, PASP, 99, 191

Stetson, P. B. 1990, PASP, 102, 932

Stetson, P. B. 1994, PASP, 106, 250

Suntzeff, N. B. 1981, ApJS, 47, 1

Sweigart, A. V., \& Mengel, J. G. 1979, ApJ, 229, 624

Trefzger, C. F., Carbon, D. F., Langer, G. E., Suntzeff, N. B., \& Kraft, R. P. 1983, ApJ, 266, 144 